\title{
Refinements and Tests of an Advanced Controller to Mitigate Fatigue Loads in the Controls Advanced Research Turbine
}

\author{
Alan D. Wright ${ }^{\dagger}$ \\ Paul Fleming $\ddagger$ \\ National Wind Technology Center \\ National Renewable Energy Laboratory ${ }^{€}$ \\ Golden, Colorado \\ Jan-Willem van Wingerden ${ }^{\epsilon €}$ \\ Delft University of Technology \\ Delft Center for Systems and Control \\ 2628 CD, Delft, The Netherlands
}

\begin{abstract}
Wind turbines are complex, nonlinear, dynamic systems forced by aerodynamic, gravitational, centrifugal, and gyroscopic loads. The aerodynamics of wind turbines are nonlinear, unsteady, and complex. Turbine rotors are subjected to a complicated 3-D turbulent wind inflow field, with imbedded coherent vortices that drive fatigue loads and reduce lifetime. Design of control algorithms for wind turbines must account for multiple control objectives. Future large multi-megawatt turbines must be designed with lighter weight structures, using active controls to mitigate fatigue loads, while maximizing energy capture. Active damping should be added to these dynamic structures to maintain stability for operation in a complex environment.

At the National Renewable Energy Laboratory (NREL), we have designed, implemented, and tested advanced controls to maximize energy extraction and reduce structural dynamic loads. These control designs are based on linear models of the turbine that are generated by specialized modeling software. In this paper, we present field test results of an advanced control algorithm to mitigate blade, tower, and drivetrain loads in Region 3. The advanced state-space controller uses independent blade pitch to mitigate the effects of shear across the rotor disk, and a collective pitch component to add active damping to the tower's first fore-aft bending mode, and, to regulate turbine speed. In addition, a separate generator torque control loop adds active damping to the tower's first side-side mode and the first drivetrain-torsion mode.

In this paper we show a refinement to the generator torque control loop to account for actuator delay. We discovered a delay in actuation between commanded generator torque and the torque actually applied to the highspeed shaft. If this delay is not properly accounted for in the plant model used for control design, the generator torque control loop tends to destabilize the first drivetrain torsion mode. We show modifications to the torque control loop to account for this delay, and, to prevent unnecessary control actuation at certain harmonics in the rotorspeed. We present field tests of this controller and make comparisons with a simple PID baseline controller for the 2-bladed Controls Advanced Research Turbine (CART2) located at NREL's National Wind Technology Center.
\end{abstract}

\footnotetext{
† Senior Engineer II, National Wind Technology Center, 1617 Cole Blvd., Mailstop 3811, AIAA member

* Engineer III, National Wind Technology Center, 1617 Cole Blvd., Mailstop 3811, AIAA member

${ }^{€}$ Employees of the Alliance for Sustainable Energy, LLC, under Contract No. DE-AC36-08GO28308 with the U.S. Dept. of Energy have authored this work. The United States Government retains, and the publisher, by accepting the article for publication, acknowledges that the United States Government retains a non-exclusive, paid-up, irrevocable, worldwide license to publish or reproduce the published form of this work, or allow others to do so, for United States Government purposes.

${ }^{€}$ Assistant Professor, Delft Center for Systems and Control, 2628 CD, Delft, AIAA nonmember
} 


\section{Introduction}

Control can improve the performance of wind turbines by enhancing energy capture and reducing dynamic loads. At the National Renewable Energy Laboratory (NREL), we are implementing and testing state-space controls on the 2-bladed Controls Advanced Research Turbine (CART2), a turbine specifically configured to test advanced controls.

Most large commercial wind turbines are variable-speed machines. In Region 2, the generator torque is controlled to extract maximum power from the wind, while blade pitch is held constant. In Region 3, generator torque is held constant, and blade pitch is used to regulate the speed of rotation of the turbine. Machines of the past used singleinput/single-output (SISO) controllers to meet these control objectives. These simple controllers, designed using Proportional Integral Derivative (PID) control, were proven effective for the rigid machines of the past, since the structural modes were well separated in frequency from the frequency band needed for the primary control objectives.

Today's trend is to design larger wind turbines with lighter weight components to reduce cost. These larger, more flexible designs may reduce the natural frequencies of important flexible modes. Now the frequencies of these structural modes might lie within the frequency band of the primary speed regulation loop. Designing the controls to properly account for these structural modes is critical. In addition, the number of objectives that the controller must meet increases to include: 1) maximizing power, 2) regulating turbine speed, 3) enhancing damping and increasing stability, and 4) reducing loads. These multi-objective controllers could be designed using the SISO classical control design methods, but this necessitates multiple control loops. The danger in this approach is that if not carefully designed, these loops can couple with each other, leading to destabilization of the turbine. Advanced multi-variable control design methods, in which all objectives are met in a very limited number of control loops, may be a better long term solution, especially as machines become lighter, larger, and more flexible.

A promising load reduction control strategy uses independent blade pitch to mitigate the effect of asymmetric wind variations across the rotor disk. ${ }^{1}$ Recently, an independent blade pitch controller (IPC) has been designed and field-tested in the NREL CART2 machine. ${ }^{2}$ This controller uses a classical control approach to design PID SISO control loops to reduce the once-per-revolution (1P) rotor loads that occur due to asymmetric shear across the disk. They also regulate speed in Region 3 and add active damping to the tower's first fore-aft mode. ${ }^{1}$ Impressive load alleviation results were demonstrated with this conventional IPC control approach. In other work, a feedback/feedforward individual pitch control method was described and tested through simulation. ${ }^{3}$ This method used an optimal multi-variable Linear Quadratic Gaussian (LQG) controller and a feed-forward disturbance rejection controller based on estimated wind speed in Region 3. This IPC approach is extended from the usual alleviation of just 1P loads, to higher harmonics $-2 \mathrm{P}$ and $3 \mathrm{P}$ load reduction. Simulation results demonstrated very good load reduction compared to the conventional IPC method in simulation studies. In another approach, two separate multivariable control loops were designed, one for the speed control and active damping of the tower fore-aft mode using collective pitch, and the other for reduction of yaw and tilt rotor moments acting on the hub. ${ }^{4}$ These two controllers were based on robust control design methods by minimizing an $\mathrm{H}_{\infty}$ norm of a weighted cost function.

In a previous paper, we showed preliminary test results from state-space controllers that perform speed regulation, load mitigation, and active damping using just two control loops. ${ }^{5} \mathrm{~A}$ independent blade pitch control loop performs Region 3 speed regulation, active damping of the tower first fore-aft (f-a) mode, and mitigation of shear across the rotor disk. The independent blade pitch control loop is designed using Disturbance Accommodating Control (DAC), including disturbance models, to account for the uniform and asymmetric wind disturbances across the rotor disk. ${ }^{6} \mathrm{~A}$ separate generator torque control loop adds active damping to the tower first side-side (s-s) mode and first drivetrain torsion mode. The generator torque control loop adds perturbations onto the normally constant Region 3 generator torque to perform this active damping. 
This paper presents a refinement of the generator torque control loop, as well as, further field-tests of this twoloop control architecture. We first discuss the turbine configuration and test set-up. We then review the pitch and generator torque control designs. We describe refinements made to the generator torque control loop to improve its performance in the presence of actuator delay, and to reduce control actuation at per revolution harmonics of the rotor-speed, where control is not needed. We then show field test results on the CART2 (Fig. 1) at the National Wind Technology Center (NWTC). We compare performance of the multi-variable state-space controller to a baseline controller based on a form of PID design.

Now, we describe the turbine for field implementation and testing.

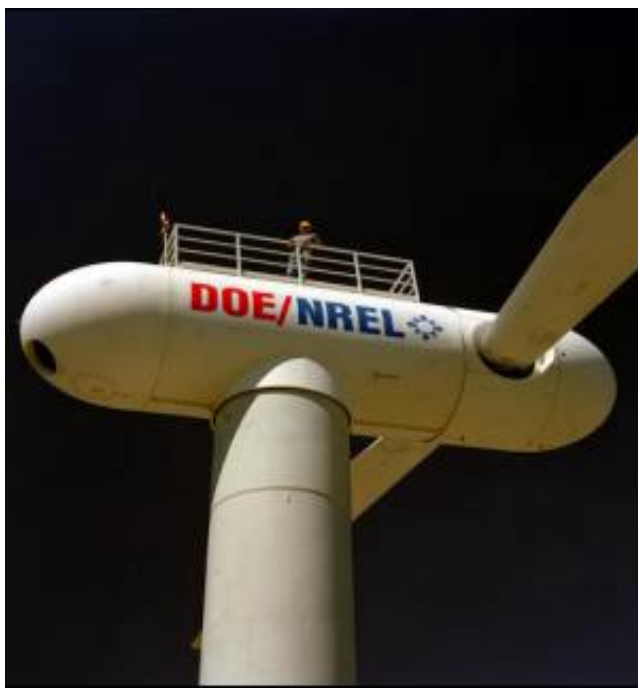

Figure 1. The two-bladed controls advanced research turbine (CART2).

\section{CART2 Configuration}

The CART2 (Fig. 1) is a two-bladed, teetered, upwind, active-yaw wind turbine. This machine is used as a test bed to study aspects of wind turbine control technology for mediumto large-scale machines. ${ }^{7}$

The CART2 is variable speed, and each blade is capable of being independently pitched with its own electromechanical servo. The pitch system can pitch the blades up to 18 degrees per second (deg/s) with pitch accelerations up to 150 degrees per second/second $\left(\mathrm{deg} / \mathrm{s}^{\wedge} 2\right)$. The squirrel cage induction generator, with full power electronics, can control torque from minus rating (motoring) to plus rating (generating) at any speed. The torque control loop has a very high rated bandwidth of 500 radians per second $(\mathrm{r} / \mathrm{s})$.

Rated electrical power (600 kilowatts at a low-speed shaft [LSS] speed of 41.7 revolutions per minute [rpm]) is maintained in Region 3 using a conventional variable-speed approach. Power electronics are used to command constant torque from the generator, and the blade pitch regulates the rotor speed.

The machine is equipped with a full complement of instruments that gather meteorological data at four heights. Blade-root flap and edge-strain gages, tower-bending gages, and low-speed shaft (LSS) and high-speed shaft (HSS) torque transducers gather load data. Accelerometers in the nacelle measure the tower's fore-aft (f-a) and side-side (ss) motion. Absolute position encoders gather data on pitch, yaw, teeter, LSS, and HSS positions. These data are sampled at $100 \mathrm{~Hz}$. The custom-built control system collects the data and controls the turbine at a control-loop cycle rate of $100 \mathrm{~Hz}$. This system is personal-computer-based and very flexible.

Next, we will review the design of the state-space controller that we field-tested.

\section{Review of Original Control Designs}

The control designs tested in this paper are based on linear time invariant (LTI) state-space control design methods. A linear time-invariant state-space model for the CART2 dynamics used for control design can be described as:

$$
\begin{aligned}
& \underline{\dot{x}}=A \underline{x}+B \underline{u}+B_{d} \underline{u}_{d} \\
& \underline{y}=C \underline{x}+D \underline{u}+D_{d} \underline{u}_{d}
\end{aligned}
$$

where $\underline{u}$ is the vector of control inputs; perturbed pitch rate of each blade or generator torque to be described shortly. The vector $\underline{u}_{d}$ is the disturbance input. $\underline{x}$ and $\underline{y}$ are the state vector and measurement vector respectively, 
described below. The matrix $A$ is the system matrix, $B$ is the control input transmission matrix, $B_{d}$ is the disturbance input transmission matrix, $C$ relates the measurements to the states, $D$ relates the measurements to the control input, and $D_{d}$ relates the measurements to the disturbance inputs. The system matrices $A, B, B_{d}, C, D$, and $D_{d}$ are found by linearizing an aeroelastic model about an operating point in Region 3 using the FatigueAerodynamics-Structures-Turbulence (FAST) code. ${ }^{8}$

This control is performed in two separate control loops, as shown in Figure 2. The independent blade pitch control loop regulates turbine speed, mitigates asymmetric wind disturbances across the rotor disk, and actively damps the tower's first fore-aft mode. The separate generator torque control loop adds active damping to the tower's first side-side mode, as well as, the drivetrain's first torsion mode.

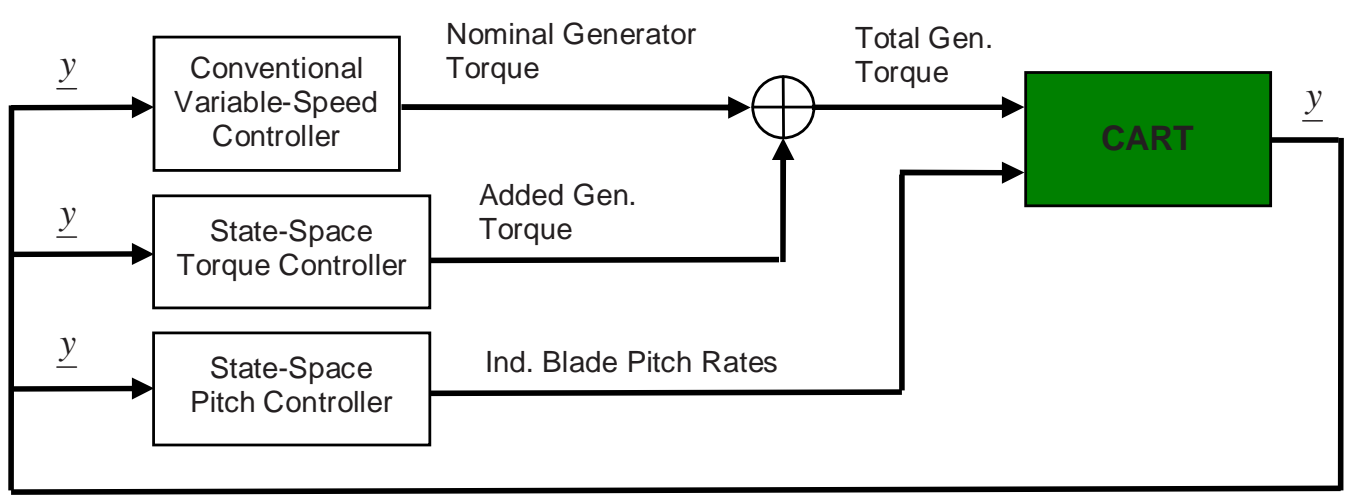

Figure 2. State-space controller architecture.

\section{Review of Independent blade pitch control loop:}

We based our independent pitch control design on Disturbance Accommodating Control (DAC). ${ }^{6}$ The basic idea of DAC is the augmentation of the usual state-estimator-based controller to recreate disturbance states via an "assumed-waveform" model. These disturbance states are used as part of the feedback control to reduce ("accommodate") or counteract any persistent disturbance effects. ${ }^{6}$ In Eq. (1), we assume a disturbance generator in the form of an additional state-space model for the wind disturbances:

$$
\begin{aligned}
& \underline{\dot{z}}_{d}(t)=F \underline{z}_{d}(t) \\
& \underline{u}_{d}(t)=\Theta \underline{z}_{d}(t),
\end{aligned}
$$

where $\underline{z}_{d}$ is the disturbance state vector, $F$ is the disturbance generator state matrix, and $\Theta$ relates the disturbance $\underline{u}_{d}(t)$ to the disturbance states. ${ }^{6}$

The objectives of the Region 3 independent blade pitch control algorithm are to regulate speed to 41.7 revolutions per minute (rpm), and to add active damping to the tower's first fore-aft bending mode, as well as to mitigate the effects of asymmetric wind disturbances across the rotor disk. As described above, the CART2 is a twobladed teetering hub machine. We will assume that the teeter degree of freedom (DOF) is locked in the design of this independent blade pitch controller, since the states corresponding to the teeter DOF are not included in the control design.

We include two wind disturbances in the vector $\underline{u}_{d}$ in Eq. (1): a disturbance representing the linear part of the wind shear, and a disturbance uniform over the rotor disk. ${ }^{7}$ The linear shear disturbance terms will induce independent blade pitch commands that will mitigate the effects of shear variations across the rotor disk. The uniform disturbance will induce rotor collective pitch commands, which act to regulate speed. A collective pitch 
component also actively damps the tower first fore-aft mode. Both the independent blade pitch and collective pitch commands are contained in the blade independent pitch loop shown in Fig. 2.

$$
\underline{u}_{d}=\left[\begin{array}{l}
u_{d_{1}}(t) \\
u_{d_{2}}(t)
\end{array}\right]=\left[\begin{array}{l}
\text { linear shear disturbance } \\
\text { uniform wind disturbance }
\end{array}\right] \text {. }
$$

The vector of plant states $\underline{x}$ for this control model can be described by:

$$
\underline{x}=\left[\begin{array}{l}
\text { perturbed tower } 1^{\text {st }} \mathrm{f} \text {-a bending deflection } \\
\text { perturbed blade } 1 \text { flapwise deflection } \\
\text { perturbed blade } 2 \text { flapwise deflection } \\
\text { perturbed tower } 1^{\text {st }} \mathrm{f} \text {-a bending velocity } \\
\text { perturbed generator speed } \\
\text { perturbed blade } 1 \text { flapwise velocity } \\
\text { perturbed blade } 2 \text { flapwise velocity } \\
\text { blade } 1 \text { perturbed pitch angle } \\
\text { blade } 1 \text { perturbed pitch rate } \\
\text { blade } 2 \text { perturbed pitch angle } \\
\text { blade } 2 \text { perturbed pitch rate }
\end{array}\right]
$$

In this plant model, extra states are included to model the effects of actuator dynamics. These states are appended to the normal plant model states, as seen in Eq. (4). The control input $\underline{u}$ consists of the perturbed pitch rate of each blade (generator torque will be the control input in a separate control loop described in the next section):

$$
\underline{u}=\left[\begin{array}{l}
\text { perturbed blade } 1 \text { pitch rate } \\
\text { perturbed blade } 2 \text { pitch rate }
\end{array}\right]
$$

We select the following five CART2 measurements:

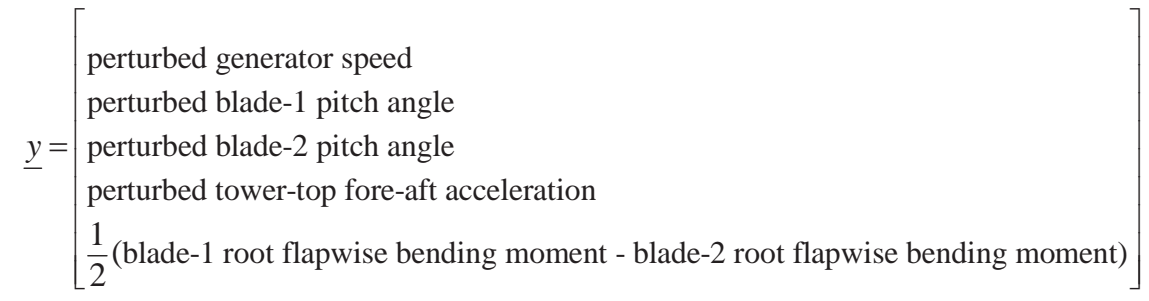

Based on these turbine measurements, state estimation is used to estimate states needed in the full state feedback control. ${ }^{7}$ These measurements provide the number necessary to provide an observable system so that state estimation can be performed. ${ }^{7}$

The controller was designed from a linear state-space model described above for the Region 3 operating point, defined by a rotor speed of $41.7 \mathrm{rpm}$, hub-height wind speed of 18.0 meters per second $(\mathrm{m} / \mathrm{s})$, and a pitch angle of 11 degrees (deg.). Reference (2) describes selection of the gains corresponding to the plant and disturbance states as well as state estimator gains.

\section{Review of Torque control loop:}

Normally, generator torque in Region 3 is held constant. In this generator torque control, perturbations in generator torque are used to add active damping to the tower first side-side (s-s) mode, as well as the first drivetrain torsion mode. We applied the state-space controls as perturbations of torque, in addition to the conventional Region 3 constant torque. This controller is designed separately from the rotor collective pitch controller using a separate linear model of the CART2. 
The state-space model of the CART2 for design of the generator torque control is described by Eq. (1), without the disturbance $\underline{u}_{d}$. For the generator torque controller, we do not need to mitigate shear or uniform wind disturbances as with the pitch controller.

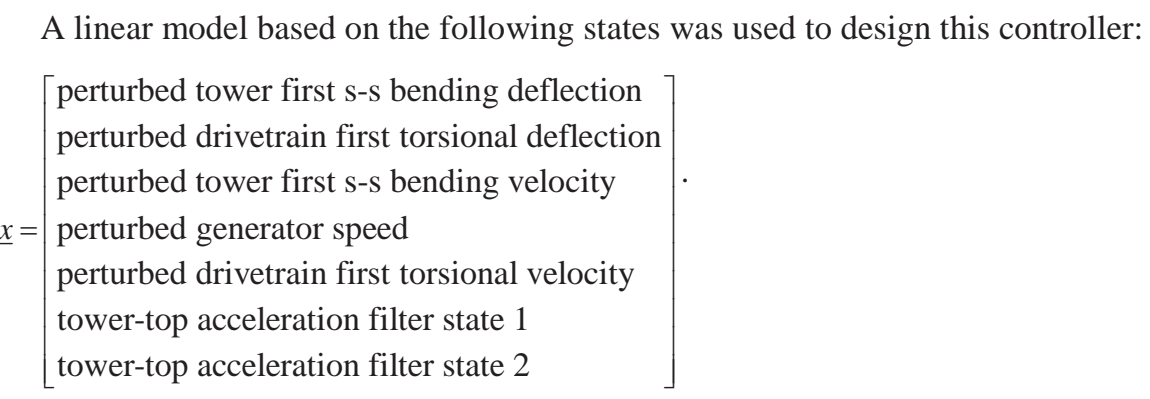

The inputs (measurements) to the state estimator controller are:

$$
\underline{y}=\left[\begin{array}{l}
\text { perturbed generator speed } \\
\text { perturbed tower-top s-s acceleration }
\end{array}\right]
$$

We added filter states to the state-space model to include a second order filter for removing high frequency noise from the tower accelerometer data. ${ }^{1}$ We again used LQR to design this controller.

Now $\underline{u}$ consists of the perturbed generator torque as the single control input for this control loop. The system matrices $(A, B, C$, and $D)$ are found by linearizing a FAST aeroelastic model at the same operating point selected for the blade pitch control design described above. The LQR state weightings were chosen to add active damping to the tower first side-side and drivetrain first torsion model. ${ }^{10}$

\section{Initial Tests:}

We implemented and began initial field tests of this two-loop, state-space controller in the winter of 2009. In the spring of 2009, the CART2 experienced a gearbox failure (caused by a gear-tooth alignment problem). The gearbox was replaced in the summer of 2009 and controls testing resumed later in the fall. Many 10-minute datasets were collected, with both the state-space controller and baseline controller, before gearbox failure and after gearbox replacement.

Testing of both controllers was performed, with the CART2 teeter degree of freedom locked, by maintaining high teeter brake pressure throughout the tests. In addition, to compare turbine loads and response between the controllers in Region 3, we extracted segments of data from each data-set that met certain criteria for Region 3 operating conditions, such as a rotor-speed above a certain limit, blade pitch angles between a certain range, etc. After these sub-sets of data were extracted, we concatenated these data together to form one long continuous dataset representing Region 3 operation for each controller. We then used $\mathrm{MCrunch}^{11}$ to calculate statistics of the data and fatigue damage equivalent loads (DELs) ${ }^{12}$ for the tower, drivetrain, and blades. 
Perhaps the best illustration of these initial field results can be seen by examining the component fatigue DELs, shown in Figure 3. Here, all results have been normalized so that the fatigue DELs for the PID baseline controller are equal to one (shown in dark blue). The green bars show the data for the state-space controller tested before gearbox failure and replacement. The red bars show the results for the same state-space controller tested after gearbox replacement. The most striking result is the state-space control results after gear replacement, which show a large increase in Low-Speed Shaft (LSS) Torque DEL (200\%). Indeed, even before gearbox failure and replacement, the state-space controller was not reducing the DEL loads in the low-speed shaft as designed, as indicated by the green bars. The statespace controller tended to reduce loads in the other components, although this performance seemed to degrade after the gearbox replacement.

A plot of the time series for Low-Speed Shaft (LSS) Torque indicated a dramatic load increase from the state-space controller after the gearbox replacement, as seen in Figure 4. Data was collected early in 2009 until the gearbox failure, which corresponded to the time series up to 700 seconds; data collected after gearbox replacement is shown after $700 \mathrm{sec}$. Figure 4 also shows a shorter section of data that compares data collected from the state-space and baseline controller after gearbox replacement. The state-space controller clearly excites the drivetrain at the first torsion mode, and does not meet the intended goal to add damping in this mode. This reflects a problem with the generator torque control design. Indeed, the generator torque controller tended to excite this mode to a lesser extent even before gearbox failure, but this problem was not discovered until after the gearbox replacement because of the dramatic increase in loads. A slight increase in the natural frequency of the actual drivetrain caused by the new gearbox may have accentuated this problem, causing the large increase in loads from the statespace controller after $700 \mathrm{sec}$.

We calculated the Power Spectral Density (PSD) of the LSS torque from each controller as seen in Figure 5. These results show peaks at 3.4 and $3.6 \mathrm{~Hz}$, which correspond to the first drivetrain torsion mode in closed-loop. The state-space controller not only increases the magnitude of this peak, but also increases its frequency, further indicating that the state-space controller is destabilizing this mode.

We investigated the cause for this loss in control performance. Upon examining CART2 test data, we discovered that a small time delay occurs between the time that generator torque is commanded by the controller and the time that this torque is actually applied to the system. This delay is present in data collected both before and after the gearbox replacement. Figure 6 shows field-test data for the CART2, illustrating a significant time delay between the commanded generator torque (demanded) and the applied torque to the High-Speed Shaft (measured). After examining several of these events, we determined that the average time delay was about $0.05 \mathrm{sec}$. The fact that the 
generator torque controller does not account for this time delay could explain the cause of the destabilization in this mode.

To examine the effects of the delay more closely, we modeled it using a first order Pade approximation. ${ }^{13}$ The phase loss at the $1^{\text {st }}$ drive train torsion frequency from this delay is approximately 58 degrees (deg.), as seen in Figure 7. To correctly add damping to the first drivetrain torsion mode, the generator torque controller must account for this phase loss.

To further study the effects of delay, we examined Bode plots of the models of the closed loop system, which consisted of the generator torque controller (before any modification to account for the delay) and linearized plant models, modified to include a 0.05 sec. delay between commanded generator torque and the actual torque input to the plant (red), seen in Figure 8. When placed in closed-loop with a plant having time delay, the generator torque controller tends to destabilize this mode, increasing the magnitude of the peak and increasing the natural frequency. This helps explain the behavior of the field test results in Figure 5, which show a shift in natural frequency and an increase in magnitude of the first drivetrain torsion mode from the state-space controller.

We also targeted another modification to the generator torque control loop. In reviewing PSD plots of generator torque from data collected from the statespace controller, we noted undesirable control actuation at frequencies corresponding to once and twice the rotor rotation rate $(1 \mathrm{P}$ and $2 \mathrm{P})$. We desired to eliminate this unnecessary control actuation. The proximity of the tower first side-side mode $(0.87 \mathrm{~Hz})$ and the $1 \mathrm{P}$ rotor frequency $(0.7 \mathrm{~Hz})$ prevented us from simply filtering the generator torque controller output. It is clear that the generator torque controller must be re-designed to include the effects of filtering to remove this actuation at $1 \mathrm{P}$ and $2 \mathrm{P}$.

We now show these generator torque control loop modifications.
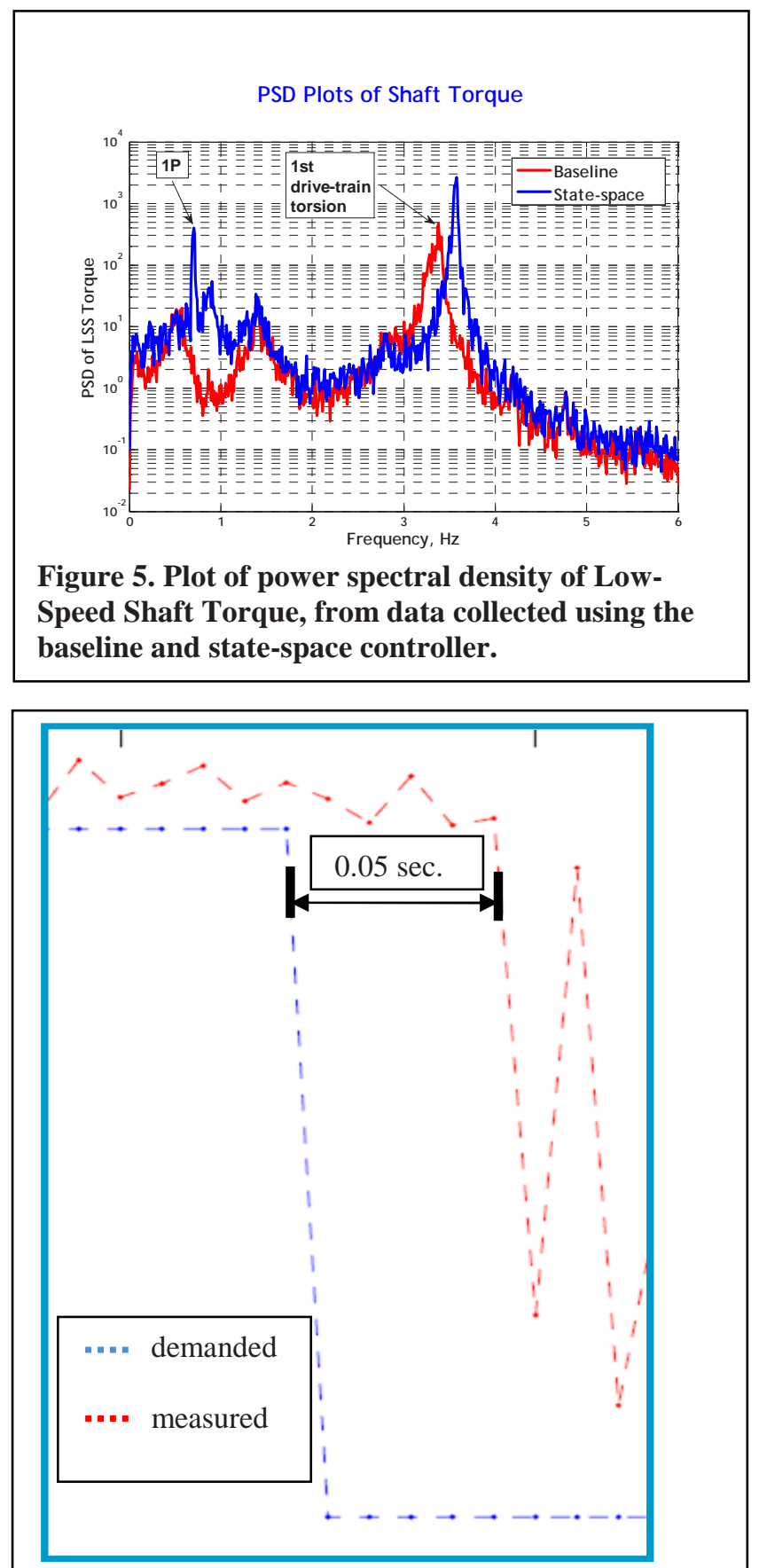

Figure 6. Plot of time series of commanded and measured generator torque showing delay.

\section{Control Design Modifications and New Test Results}

The generator torque controller was re-designed to include the effects of the time delay and to eliminate unnecessary control actuation at $1 \mathrm{P}$ and $2 \mathrm{P}$. The re-design to account for delay consisted of augmenting the linear plant state-space model, with a single state representing the delay, from the Pade approximation model. ${ }^{13}$

In addition, we refined the generator torque control design to reduce generator torque control actuation at the $1 \mathrm{P}$ and $2 \mathrm{P}$ rotor harmonics. We followed an approach that adds frequency-dependent state and control weighting to the 
standard LQR optimal control problem. ${ }^{14}$ Using frequency shaped cost functionals, a control designer may explicitly shape the loop dynamics, by selecting state and/or control weighting functions. To reduce control actuation at $1 \mathrm{P}$ and $2 \mathrm{P}$, we penalized control actuation at these frequencies using inverted notch filters centered at these two frequencies. We calculated the full state feed-back and state estimator gain matrices by casting this augmented system as an $\mathrm{H} 2$ optimization problem and solving the resulting matrix Riccati equations in MATLAB. ${ }^{15}$

We implemented the revised generator torque control loop in the CART2 and resumed field tests. We again tested both the revised state-space controller and the baseline controller. MCrunch again was used to calculate statistics and DEL fatigue loads from the test data. Table 2 shows statistics of the results, from both the baseline controller and the revised state-space controller. Figure 9 shows component load fatigue DELs, comparing the performance of the revised statespace, original state-space, and baseline controllers. Of significance is the large reduction in the Low-speed Shaft Torque DEL, with the revised state-space controller (statespace new). This load reduction reflects the redesign to account for the effects of the generator torque time delay. It also reflects the modified LQR design, with frequency weighting on the control output at the $1 \mathrm{P}$ and $2 \mathrm{P}$ rotor harmonics - preventing unnecessary control actuation at those frequencies. Preventing this unnecessary control actuation also reduces torque loads that the generator applies to the drivetrain at $1 \mathrm{P}$ and $2 \mathrm{P}$.
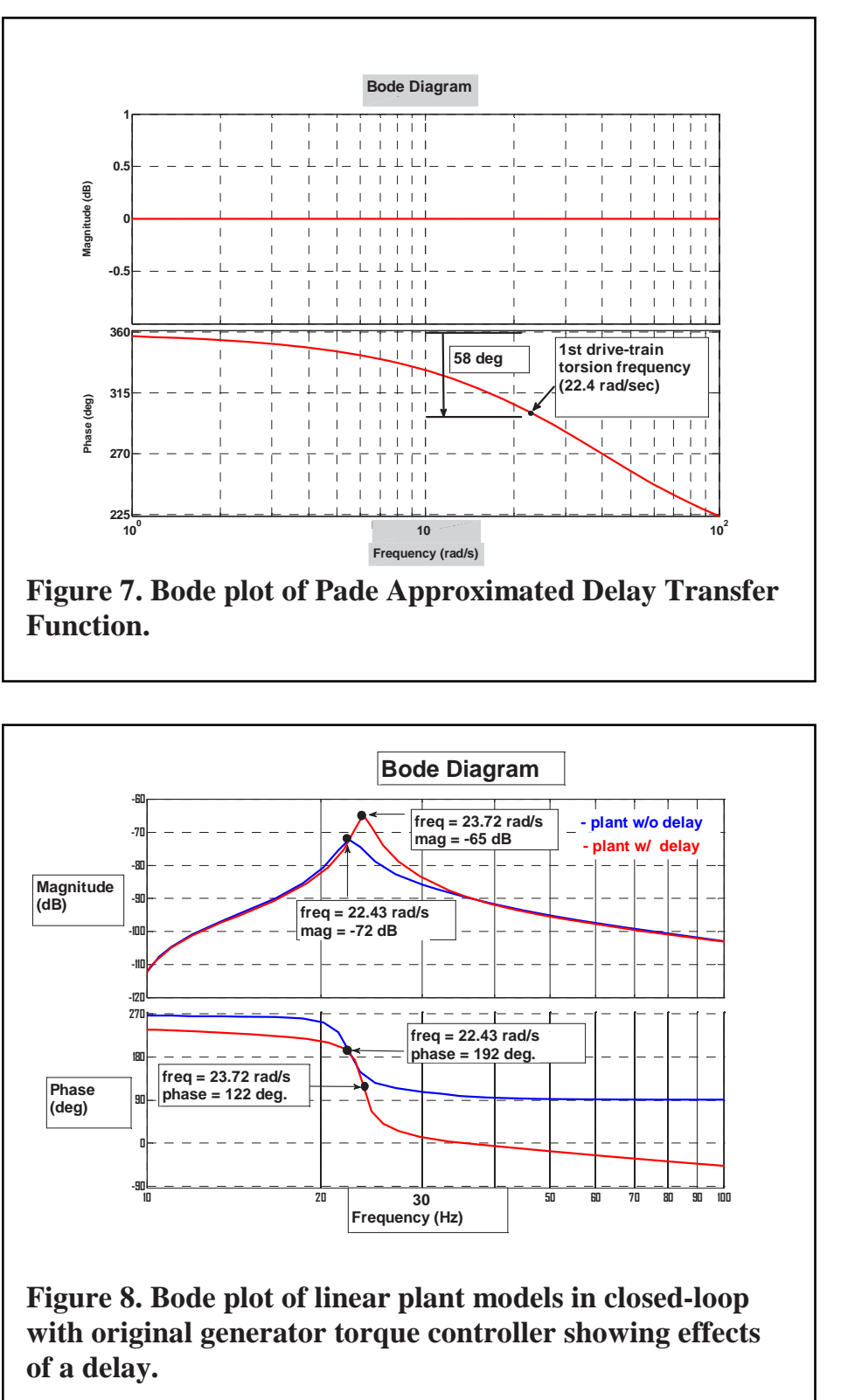

We also compared psd's of the Low-speed Shaft Torque from the baseline and revised state-space controllers, as shown in Figure 10. This figure shows a dramatic reduction in the magnitude of the peak at the first drivetrain torsional frequency, from the revised state-space controller, showing that active damping is successfully being applied to this mode using generator torque control. 
TABLE 2. COMPARISON OF BASELINE AND

StATE-SPACE CONTROLlER (BLADE INDEPENDENT PITCH AND GENERATOR TORQUE) DATASET STATISTICS FOR REGION 3 OPERATION (LOCKED TEETER)

\begin{tabular}{|c|c|c|}
\hline $\begin{array}{c}\text { Statistics and } \\
\text { Performance Measure }\end{array}$ & $\begin{array}{l}\text { Baseline } \\
\text { PID Control }(1460 \mathrm{sec} .)\end{array}$ & $\begin{array}{c}\text { Revised State-Space } \\
(1220 \mathrm{sec} .)\end{array}$ \\
\hline Wind speed $(\mathrm{m} / \mathrm{s})$ & $\begin{array}{l}\text { mean } 14.92 \\
\text { std } 2.33\end{array}$ & $\begin{array}{l}\text { mean } 15.83 \\
\text { std } 1.90\end{array}$ \\
\hline Rotor speed $(\mathrm{rpm})$ & $\begin{array}{l}\text { mean } 41.61 \\
\text { std } 0.44\end{array}$ \\
\hline Blade pitch $(\mathrm{deg})$ & mean 6.21 & mean 9.06 \\
& std 4.18 & std 2.02 \\
\hline Blade pitch rate $(\mathrm{deg} / \mathrm{s})$ & std 2.1 & std 3.2 \\
& $\max 13.9$ & $\max 14.9$ \\
& $\min -11.4$ & $\min -15.3$ \\
\hline Generator torque $(\mathrm{kNm})$ & Std 158.4 124.8 \\
& Max 3524 & $\max 4002$ \\
& $\min 2526$ & $\min 3048$ \\
\hline
\end{tabular}

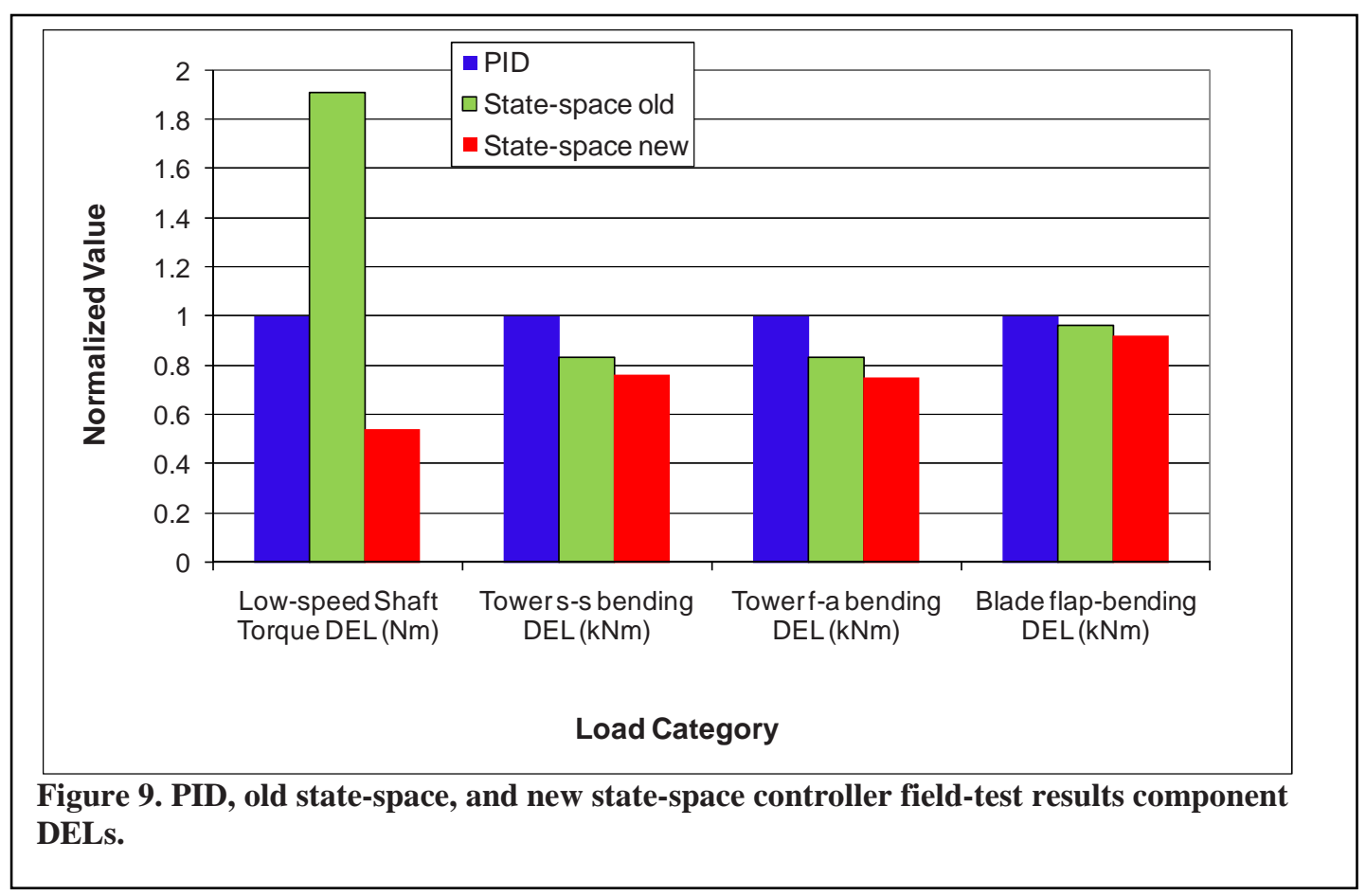

The revised state-space controller also reduces loads in the other components, as seen in the red bars in Figure 9. The blade pitch controller is reducing tower fore-aft fatigue DELs by adding active damping to the tower's first fore-aft mode. The generator torque controller is successfully reducing tower side-side fatigue DELS through active damping of the tower's first side-side mode. The independent blade pitch is reducing the blade flap-bending fatigue DEL's through mitigation of the effects of shear across the rotor disk. This is a result of including a model of the shear disturbance into the augmented plant model used for the design of the independent blade pitch controller. All of the objectives in blade pitch control are accomplished, with just a modest increase in blade pitch rates, compared to the baseline controller, as seen in Table 1 (blade pitch rates). 

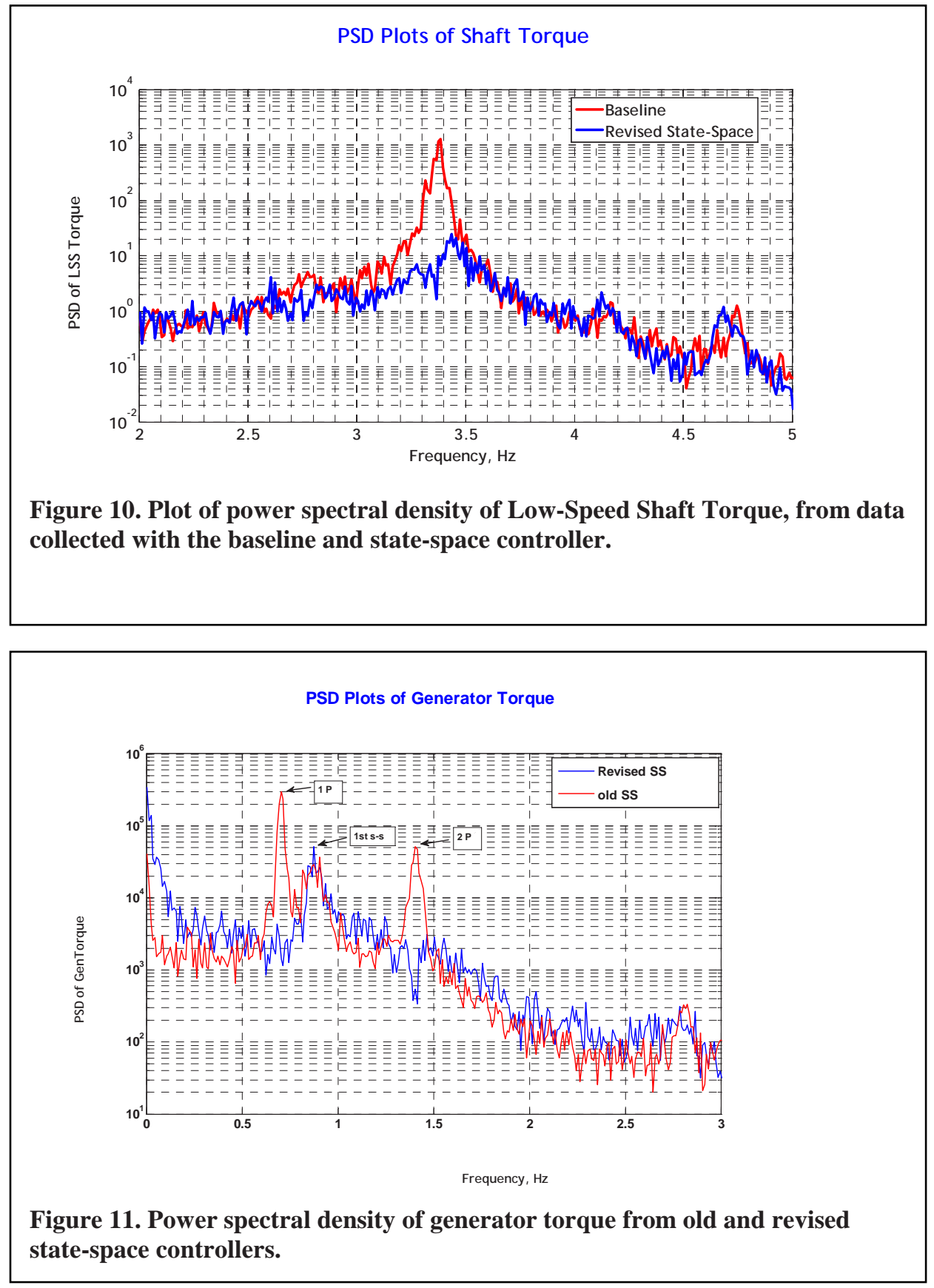

In Figure 11, we see that the new generator torque state-space controller eliminates control actuation at 1P and $2 \mathrm{P}$, when compared to the old generator torque state-space controller. Both controllers provide actuation at the tower's first side-side (s-s) mode, meeting the control objective of adding damping to this very lightly damped structural mode. Elimination of actuation at these per-revolution harmonics of rotor-speed also contributes to the fatigue DEL reduction, from the revised controller, seen in Figure 10.

\section{Conclusions}

A two-loop control architecture for mitigating wind turbine fatigue loads in Region 3 has been described. The blade pitch control loop is designed to regulate turbine speed and add active damping to the tower's first fore-aft mode, with a collective pitch component, and to mitigate the effects of asymmetric wind distributions across the 
rotor, with an independent blade pitch component. A separate generator torque control loop adds active damping to the tower's first side-side mode, as well as, to the drivetrain's first torsion mode. The blade pitch control algorithm is designed using Disturbance Accommodating Control, wherein a model of the disturbance using disturbance waveforms is included in the linear model for control design.

After implementation and the initial field tests of this advanced controller, the CART2 machine gearbox failed due to a gear tooth misalignment. After replacement of this gearbox with a spare, field tests were resumed. The generator torque control loop seemed to increase drivetrain torque loads dramatically, when compared to the limited data gathered before the gearbox failure. The cause of this instability was determined to be a small time delay ( 0.05 sec.) between the time that the generator torque was demanded and application of the torque to the real plant. The original generator torque control loop did not account for this delay.

The generator torque control loop was re-designed to account for this delay, as well as, to reduce unnecessary control actuation at certain harmonics in the rotor-speed. Initial field tests of the re-designed controller demonstrated improved control performance due to these modifications. Initial field tests demonstrated that the two loop blade pitch controller and generator torque controller successfully reduced fatigue loads in the Low-speed shaft, blade roots, and tower.

Initial test results, with the state-space controller, show promise for the load mitigation capability of this controller, compared to a standard baseline PID controller in Region 3 turbine operation. Further testing of this revised state-space controller is needed to further validate the performance of this controller. Additional tuning of the gains may be attempted to obtain further load reductions, especially in the blade flap-bending loads, by tuning of the shear disturbance gain. These gain increases will have to be balanced, with the resulting increase in blade pitch rates. We believe that more significant blade load reductions may be possible through increasing this disturbance gain.

\section{Future Work}

We plan to continue field-tests of this two loop control architecture on the NREL CART2 machine. These final tests will conclude the work on this two-loop advanced controller and the results will be documented through a final report.

\section{References}

\footnotetext{
${ }^{1}$ Bossanyi, E., "Developments in Individual Blade Pitch Control," EWEA Conference, The Science of Making Torque from Wind, DUWIND, Delft University of Technology, The Netherlands, April 19-21, 2004.

${ }^{2}$ Bossanyi, E., Wright, A., and Fleming, P., "Controller field tests on the NREL CART2 turbine," UPWIND report 11593/BR/08, 22 $2^{\text {nd }}$ June 2010, available at http://www.upwind.eu/Shared\%20Documents/WP5\%20\%20Publications/D\%205.6.1.pdf.

${ }^{3}$ van Engelen, T.G., "Design Model and Load Reduction Assessment for Multi-rotational Mode Individual Pitch Control," Presented at the 2006 European Wind Energy Conference and Exhibition, Athens, Greece, 27 February - 2 March, 2006.
}

${ }^{4}$ Geyler, M., and Caselitz, P., "Individual Blade Pitch Control Design for Load Reduction on Wind Turbines," Presented at the 2007 European Wind Energy Conference and Exhibition, Milan, Italy, 7-10 May, 2007.

${ }^{5}$ Wright, A., Fingersh, L., and Stol, K., "Testing Further Controls to Mitigate Loads in the Controls Advanced Research Turbine,” 29th ASME Wind Energy Conference, Orlando, Fl., January 2010.

${ }^{6}$ Balas, M.J., Lee, Y.J., and Kendall, L., "Disturbance Tracking Control Theory with Application to Horizontal Axis Wind Turbines," 17th ASME Wind Energy Conference, Reno, Nevada, 12-15 January, 1998.

${ }^{7}$ Fingersh, L., and Johnson, K., "Baseline Results and Future Plans for the NREL Controls Advanced Research Turbine," $23^{\text {rd }}$ ASME Wind Energy Conference, Reno, NV, 2004, pp. 87-93.

${ }^{8}$ Jonkman, J. M., and Buhl, M. L., "FAST User's Guide," National Renewable Energy Laboratory, NREL/EL-500-38230, Golden, CO, 2005.

${ }^{9}$ Wright, A. D., "Modern Control Design for Flexible Wind Turbines," National Renewable Energy Laboratory, NREL/TP500-39154, Golden, CO, 2004 at http://www.nrel.gov/docs/fy06osti/39154.pdf. 
${ }^{10}$ Kwakernaak, H., and Sivan, R., Linear Optimal Control Systems, Wiley Interscience, NY, 1972, pp. $207-237$.

${ }^{11}$ Buhl, M. L. Jr.., “MCrunch User's Guide for Version 1.00,” NREL Report No. TP-500-43139, National Renewable Energy Laboratory, Golden, Colorado, May, 2008.

${ }^{12}$ Sutherland, H. J., "On the Fatigue Analysis of Wind Turbines," Sandia National Laboratories, SAND99-0089, Albuquerque, New Mexico, June 1999.

${ }^{13}$ Baker, G. A. Jr. "The Theory and Application of The Pade Approximant Method." In Advances in Theoretical Physics, Vol. 1 (Ed. K. A. Brueckner). New York: Academic Press, pp. 1-58, 1965.

${ }^{14}$ Gupta, N. K., "Frequency-Shaped Cost Functionals: Extension of Linear-Quadratic-Gaussian Design Methods," Journal of Guidance and Control, Vol. 3, No. 6, Nov.-Dec. 1980.

${ }^{15}$ Skogestad, S., and Postlethwaite, I., Multivariable Feedback Control Analysis and Design, John Wiley \& Sons, Ltd, West Sussex, England, 2005. 